XLVIII.-The Residual Affinity of the Coumarins and Thiocoumarins as shown by their Additive Compounds.

By Arthur Clayton, B.Sc.

Coumarin, the lactone of o-coumaric acid, possesses in a marked degree the property of yielding additive compounds with reagents of very diverse types, and in this respect is distinguished from the lactones in general, which do not as a class exhibit this feature to any great extent.

The earliest known additive compound of coumarin was the dibromide discovered by Sir W. H. Perkin (Annalen, 1871, 157. 116), and afterwards again examined by Ebert (Annalen, 1884, 226, 348). The results obtained by these investigators render it certain that the bromine is attached, not to the carbon atoms united by unsaturated linking, but either to the oxidic or the ketonic oxygen. This is shown by the ease with which the halogen is entirely eliminated. If the ordinary lactonic constitution for coumarin be accepted, this dibromide might be represented by formula $I$, whilst the alternative configuration suggested by Morgan and Micklethwait (Trans., 1906, 87, 868) would lead to formula II :

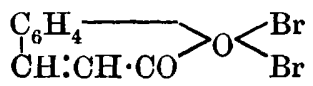

I.

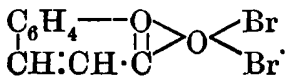

II.

Coumarin combines additively with the metallic oxides and hydroxides, as was shown long ago by $\mathrm{R}$. Williamson (Jahresber., 1875, 587 ), these compounds generally conforming to the types $\mathrm{C}_{9} \mathrm{H}_{6} \mathrm{O}_{2}, 2 \mathrm{ROH}$ and $\mathrm{C}_{9} \mathrm{H}_{8} \mathrm{O}_{2}, \mathrm{R}_{2} \mathrm{O}$, where $\mathrm{R}$ is an alkali metal. The next observation of the combining proclivities of coumarin is due to Ebert, who found (loc. cit.) that the lactone dissolved easily in concentrated hydrobromic acid, the solution furnishing a hydrobromide, which, however, was so unstable that it could only be preserved at low temperatures, and was therefore not analysed.

By employing concentrated hydrochloric acid as the solvent and then adding a strong solution of chloroplatinic acid, Morgan and 
Micklethwait (ibid, p. 863) obtained arew Article Online $4 \mathrm{C}_{9} \mathrm{H}_{6} \mathrm{O}_{2}, \mathrm{H}_{2} \mathrm{PtCl}_{6}, 4 \mathrm{H}_{2} \mathrm{O}$, to which they gave the graphic formula

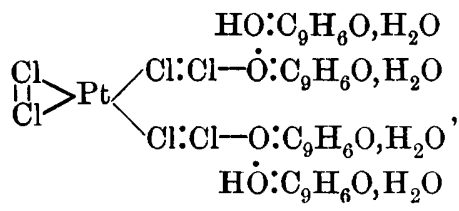

where $\mathrm{C}_{9} \mathrm{H}_{6} \mathrm{O}$ is the coumarin residue, excluding the oxygen atom, which becomes quadrivalent in the formation of the double salt. These authors (loc. cit.) also obtained a cobalticyanide of a more normal type, which has a composition corresponding with the formula $3 \mathrm{C}_{9} \mathrm{H}_{6} \mathrm{O}_{2}, \mathrm{H}_{3} \mathrm{Co}(\mathrm{CN})_{6}, 3 \mathrm{H}_{2} \mathrm{O}$.

In the experiments described in this paper, the investigation has been extended to the homologues and the sulphur analogues of coumarin, and in most cases it has been found possible to demonstrate the existence of additive compounds, either of the oxonium types or produced by the union of the lactone itself with a metallic salt.

\section{Coumarin and Thiocoumarin.}

Coumarin Mercurichloride.-Coumarin and mercuric chloride in equimolecular proportion were dissolved in the smallest possible quantity of ether and mixed together. On leaving the mixture for one or two days, the mercurichloride separated in long, colourless needles which melted at $164-165^{\circ}$ :

0.8590 gave $0.5782 \mathrm{AgCl}$. $\mathrm{Cl}=16.65$.

$0.6381,03712 \mathrm{HgS}$. $\mathrm{Hg}=46.84$.

$\mathrm{C}_{9} \mathrm{H}_{6} \mathrm{O}_{2} \mathrm{Cl}_{2} \mathrm{Hg}$ requires $\mathrm{Cl}=17.03 ; \mathrm{Hg}=47.96$ per cent.

Recrystallisation was not found to be advisable, as the affinity between the generators is so slight that dissociation readily occurs. As an instance of the feebleness of the union, it was found that aniline mercurichloride slowly separated on mixing ethereal solutions of aniline and coumarin mercurichloride.

Thiocoumarin, prepared by Tiemann's method (Ber., 1886, 19, 1661), is generally obtained in dark brown needles, rather difficult to purify by recrystallisation. The substance can, however, readily be purified by boiling its benzene solution for several hours with copper powder and subsequently crystallising from alcohol, when it possesses a brilliant, golden-yellow colour. This method of purification increases the yield of pure thiocoumarin from 50 to 80 per cent. (compare Tiemann, loc. cit.).

Thiocoumarin mercurichloride was prepared by mixing an equimolecular proportion of thiocoumarin and mercuric chloride in ethereal 
solution. A yellow powder immediately separated, and Article Online became quite colourless. The mercurichloride is very insoluble in ordinary solvents, boiling nitrobenzene being the only liquid which appears to exert any solvent action. The substance is decomposed when suspended in water and treated with hydrogen sulphide, thiocoumarin being liberated. On analysis, the mercurichloride gave numbers approximating to those required by the formula

$$
\mathrm{C}_{9} \mathrm{H}_{6} \mathrm{OS}, \mathrm{HgCl}_{2} \text {. }
$$

\section{7-Methylcoumarin and 7-Methylthiocoumarin.*}

7-Methylcoumarin Mercurichloride.-Whe combination of the generators of this substance resulted from their admixture in ethereal solution. As in the case of coumarin mercurichloride some time elapses before crystallisation sets in, attempts at rapid crystallisation always resulting in the separation of an impure product. When once the compound has been prepared, however, crystallisation may be effected much more rapidly. It forms long, colourless needles soluble in ether, benzene, chloroform, and to some extent in water, and from each of these solvents the substance may be recovered unchanged, but, being even more unstable than coumarin mercurichloride, recrystallisation was not found useful as a means of purification. The crystals first formed melted very definitely at $170^{\circ}$ :

$$
\begin{aligned}
& 0.4099 \text { gave } 0.2575 \mathrm{AgCl} \text {. } \mathrm{Cl}=15.54 . \\
& \qquad \mathrm{C}_{10} \mathrm{H}_{8} \mathrm{O}_{2} \mathrm{Cl}_{2} \mathrm{Hg} \text { requires } \mathrm{Cl}=16.47 \text { per cent. }
\end{aligned}
$$

7-Methylcoumarin Cobalticyanide.-One gram of 7-methylcoumarin was dissolved in concentrated hydrochloric acid; the solution was cooled as rapidly as possible to prevent crystallisation, and then added to an excess of cobalticyanic acid prepared according to von Baeyer and Villiger's directions (Ber., 1901, 34, 2687). The compound separated as a white, crystalline powder, and after several days the mother liquor yielded colourless cubes of the same substance. After filtration, the powder was washed with dilute hydrochloric acid, and dried at the ordinary temperature as water of crystallisation was evolved at $100^{\circ}$ :

0.6456 gave $0.1058 \mathrm{H}_{2} \mathrm{O} . \quad \mathrm{H}_{2} \mathrm{O}=16.39$.

$0.8030,0.1118 \mathrm{Co} . \quad \mathrm{Co}=13.92$. $\mathrm{C}_{10} \mathrm{H}_{8} \mathrm{O}_{2}, \mathrm{H}_{3} \mathrm{Co}(\mathrm{CN})_{6}, 4 \mathrm{H}_{2} \mathrm{O}$ requires $\mathrm{H}_{2} \mathrm{O}=16.00 ; \mathrm{Co}=13.11$ per cent.

7-Methylcoumarin platinichloride was not prepared in the pure state,

* In this paper the notation is in accordance with the numbering indicated in the formula 
but on mixing concentrated solutions of 7-methylcoumarin and chloroplatinic acid in concentrated hydrochloric acid, yellow crystals containing platinum separated. These were instantly decomposed by moisture.

7-Methylttiocoumarin.-The preparation of this compound was effected by heating an intimate mixture of equal weights of 7-methylcoumarin and phosphorus pentasulphide to $120^{\circ}$ in a paraffin-bath until the mixture had been molten for seven minutes. The powdered mass, after extraction with benzene and subsequent crystallisation from alcohol, was treated with copper powder, and then appeared as glistening, golden-yellow leaflets melting at $125-126^{\circ}$ :

0.1664 gave $0.2243 \mathrm{BaSO}_{4}$. $\mathrm{S}=18.51$.

$\mathrm{C}_{10} \mathrm{H}_{8} \mathrm{OS}$ requires $\mathrm{S}=18 \cdot 18$ per cent.

On shaking a solution of the thiocoumarin in benzene with yellow mercuric oxide, 7-methylcoumarin was regenerated, thus proving the constitution of the substance.

7-Methylcoumarinoxime.-This substance was obtained by heating equal molecules of sodium hydroxide, 7-methylthiocoumarin, and hydroxylamine hydrochloride in alcoholic solution for two days. The product, when poured into water, yielded an emulsion which soon furnished the solid oxime. This was collected and crystallised from boiling water, from which it separated in white needles melting at $178^{\circ}$ :

0.2664 gave $17 \cdot 85$ c.c. nitrogen at $20^{\circ}$ and $773 \mathrm{~mm} . \quad \mathrm{N}=7 \cdot 77$.

$\mathrm{C}_{10} \mathrm{H}_{9} \mathrm{O}_{2} \mathrm{~N}$ requires $\mathrm{N}=8.00$ per cent.

The substance is less soluble in water than coumarinoxime.

7-Methylcoumarinphenylhydrazone.-An alcoholic solution of equal molecules of 7-methylthiocoumarin and phenylhydrazine was boiled until no more hydrogen sulphide was evolved, the operation requiring about four days. On cooling the solution, yellowish-brown needles were obtained, which, after three crystallisations from alcohol, melted at $139^{\circ}$ :

0.2635 gave $25 \cdot 8$ c.c. nitrogen at $20^{\circ}$ and $773 \mathrm{~mm}$. $\quad \mathrm{N}=11.36$.

$\mathrm{C}_{16} \mathrm{H}_{14} \mathrm{ON}_{2}$ requires $\mathrm{N}=11 \cdot 20$ per cent.

7-Methylcoumarin mercurichloride, prepared in the same way as coumarin mercurichloride, was obtained as a yellow powder very similar to the latter substance:

0.5207 gave $0.3439 \mathrm{AgCl}$. $\mathrm{Cl}=16.34$.

$\mathrm{C}_{10} \mathrm{H}_{8} \mathrm{OSCl}_{2} \mathrm{Hg}$ requires $\mathrm{Cl}=15.88$ per cent.

The compound decomposes without melting, and regenerates 7-methylthiocoumarin when treated with hydrogen sulphide. 


\section{4 : 7-Dimethylcoumarin and 4 : 7-Dimethylthiocoumarin.}

4:7-Dimethylcoumarin Mercurichloride. -The combination of mercuric chloride and 4:7-dimethylcoumarin was effected by a method similar to that used for the preparation of 7-methylcoumarin mercurichloride. The substance, which was produced more readily and more plentifully than the latter compound, forms beautiful, white needles, crystals two inches and more in length being easily obtained. Its behaviour towards solvents is very similar to that of 7-methylcoumarin mercurichloride, and although more stable than this compound it appears in its purest form before crystallisation, when it melts very definitely at $175^{\circ}$. Two or three crystallisations did not noticeably change the melting point:

$$
\begin{aligned}
& 0.7226 \text { gave } 0.4414 \mathrm{AgCl} . \mathrm{Cl}=15 \cdot 12 \text {. } \\
& 0.4855,0.2456 \mathrm{HgS} \text {. } \quad \mathrm{Hg}=43.61 \text {. } \\
& \mathrm{C}_{11} \mathrm{H}_{10} \mathrm{O}_{2} \mathrm{Cl}_{2} \mathrm{Hg} \text { requires } \mathrm{Cl}=15.95 ; \mathrm{Hg}=44.94 \text { per cent. }
\end{aligned}
$$

4:7-Dimethylcoumarin cobalticyanide was obtained by adding a solution of 4:7-dimethylcoumarin in concentrated hydrochloric acid to an excess of cobalticyanic acid in the same solvent. The white, microcrystalline product was much more easily and plentifully obtained than the corresponding compound of 7-methylcoumarin. As it could not be recrystallised without decomposition, the substance was washed with dilute hydrochloric acid and dried. It was found to lose weight at $100^{\circ}$, and in order to prove that this loss was due to the elimination of water and not to the evolution of gases containing nitrogen, the cobalt was estimated in the substance before heating and the nitrogen after three hours' heating in a steam-oven :

0.8784 gave $0.0908 \mathrm{H}_{2} \mathrm{O} . \quad \mathrm{H}_{2} \mathrm{O}=15.72$.

$0.6331,0.0815$ Co. Co=12.87. $\mathrm{C}_{11} \mathrm{H}_{10} \mathrm{O}_{2}, \mathrm{H}_{3} \mathrm{Co}(\mathrm{CN})_{6}, 4 \mathrm{H}_{2} \mathrm{O}$ requires $\mathrm{H}_{2} \mathrm{O}=15.52 ; \mathrm{Co}=12.72$ per cent.

0.2103 of the anhydrous substance gave 40.0 c.c. nitrogen at $21^{\circ}$ and $757 \mathrm{~mm} . \mathrm{N}=21 \cdot 64$.

$$
\mathrm{C}_{11} \mathrm{H}_{10} \mathrm{O}_{2}, \mathrm{H}_{3} \mathrm{Co}(\mathrm{CN})_{6} \text { requires } \mathrm{N}=21.43 \text { per cent. }
$$

$4: 7$-Dimethylcoumarin platinichloride was not obtained in the pure state, but yellow crystals were formed which melted indefinitely between $160^{\circ}$ and $170^{\circ}$, and on analysis were found to contain 22.5 per cent. of platinum. The oxonium salt was immediately decomposed by water, and even by concentrated hydrochloric acid, with the liberation of 4:7-dimethylcoumarin, but was recrystallised from a concentrated solution of platinic chloride in the latter solvent. Since it was not found possible to wash the crystals without decomposing them, data for the establishment of a formula could not be obtained. 
4: 7-Dimethylthiocoumarin resulted when $4: 7$-dimethylthiocoumarin was subjected to the same treatment as that employed for the conversion of 7-methylcoumarin into its sulphur analogue; it crystallised from alcohol in dark needles which, after eleven crystallisations, were obtained with a greenish-yellow colour, which was not noticeably changed by treatment with copper powder. Like its homologues, it yields the corresponding coumarin when treated with yellow mercuric oxide. The pure substance melts at $118-119^{\circ}$ :

$0 \cdot 3045$ gave 0.3789 $\mathrm{BaSO}_{4} . \quad \mathrm{S}=17 \cdot 09$.

$$
\mathrm{C}_{11} \mathrm{H}_{10} \mathrm{OS} \text { requires } \mathrm{S}=16.80 \text { per cent. }
$$

It was noticed throughout that there was a strong tendency for the thiocoumarins to give on analysis a high percentage for sulphur, and that this extra sulphur was retained with considerable persistency through several successive crystallisations. This property was especially marked in the case of $4: 7$-dimethylthiocoumarin, which after three crystallisations from alcohol yielded 21 per cent. of sulphur, thus making it appear likely that the sulphur is united to the thiocoumarin by some weak affinity. These loosely combined sulphur compounds can generally be removed by the treatment with copper powder mentioned on page 525 .

$4: 7$-Dimethylcoumarinoxime was obtained by the same method as that used for the preparation of 7 -methylcoumarinoxime; it is less soluble in water than the latter compound, but separates from a mixture of alcohol and water in fine, white needles melting at $179^{\circ}$ :

0.3377 gave $21 \cdot 4$ : c.c. nitrogen at $19^{\circ}$ and $748 \mathrm{~mm}$. $N=7 \cdot 17$.

$$
\mathrm{C}_{11} \mathrm{H}_{11} \mathrm{O}_{2} \mathrm{~N} \text { requires } \mathrm{N}=7 \cdot 41 \text { per cent. }
$$

4 : 7-Dimethylcoumarinphenylhydrazone, prepared in the same manner as 7-methylcoumarinphenylhydrazone, forms golden-brown needles melting at $99-100^{\circ}$ :

$0 \cdot 1947$ gave $18 \cdot 8$ c.c. nitrogen at $22^{\circ}$ and $762 \mathrm{~mm}$. $\quad \mathrm{N}=10 \cdot 97$. $\mathrm{C}_{17} \mathrm{H}_{16} \mathrm{ON}_{2}$ requires $\mathrm{N}=10 \cdot 61$ per cent.

4:7-Dimethylthiocoumarin Mercurichloride.-The addition of the generators of this sabstance is effected by their admixture in ethereal solution. The product forms a yellow, amorphous powder with great readiness, and behaves towards solvents like thiocoumarin mercurichloride. Hydrogen sulphide regenerates $4: 7$-dimethylthiocoumarin :

0.1466 gave $0.0934 \mathrm{AgCl} . \mathrm{Cl}=15 \cdot 78$.

$$
\mathrm{C}_{11} \mathrm{H}_{10} \mathrm{OSCl}_{2} \mathrm{Hg} \text { requires } \mathrm{Cl}=15 \cdot 40 \text { per cent. }
$$

\section{$3: 4: 7$-Trimethylcoumarin and $3: 4$ - 7-Trimethylthiocoumarin.}

$3: 4: 7$-Trimethylcoumarin mercurichloride, obtained by the general method of preparation, separated from ether in a rather ill-defined, 
felt-like mass, totally different in appearance from the View Article Online chlorides. Analysis gave numbers which approximated to those required by the formula $\mathrm{C}_{12} \mathrm{H}_{12} \mathrm{O}_{2}, \mathrm{HgCl}_{2}$.

3:4:7-Trimethylcoumarin cobalticyanide was obtained with some difficulty by the method already employed, and presented the same features as the other cobalticyanides, being dificult to obtain in a state approaching purity. Data were obtained which indicated the formula $\mathrm{C}_{12} \mathrm{H}_{12} \mathrm{O}_{2}, \mathrm{H}_{3} \mathrm{Co}(\mathrm{CN})_{6}$ for the anhydrous substance.

$3: 4: 7$-Trimethylcoumarin platinichloride was not prepared in the pure state, but indications of its existence were obtained.

$3: 4: 7$-Trimethylthiocoumarin.-The preparation of this substance proceeded smoothly by the general method, the compound being finally obtained in very brilliant reddish-yellow needles melting at $124-125^{\circ}$ :

0.3114 gave $0.3731 \mathrm{BaSO}_{4} . \quad \mathrm{S}=16.46$.

$\mathrm{C}_{12} \mathrm{H}_{12} \mathrm{OS}$ requires $\mathrm{S}=15 \cdot 69$ per cent.

The product has the general properties and solubilities of the thiocoumarins.

3:4:7-Trimethylcoumarinoxime results from the interaction of equimolecular proportions of $3: 4: 7$-trimethylthiocoumarin, sodium hydroxide, and hydroxylamine hydrochloride. It is much less soluble than 4:7-dimethylcoumarinoxime, but crystallises from alcohol in needles melting at $198^{\circ}$ :

0.2099 gave 11.4 c.c. nitrogen at $9^{\circ}$ and $770 \mathrm{~mm}$. $\mathrm{N}=6.61$. $\mathrm{C}_{12} \mathrm{H}_{13} \mathrm{O}_{2} \mathrm{~N}$ requires $\mathrm{N}=6.89$ per cent.

$3: 4: 7$-Trimethylcoumarinphenylhydrazone was obtained in beautiful yellow needles by the general method of preparation. It may be crystallised from alcohol, and melts at $135^{\circ}$ :

0.2020 gave 18.3 c.c. nitrogen at $19^{\circ}$ and $733 \mathrm{~mm}$. $\mathrm{N}=10.02$. $\mathrm{C}_{18} \mathrm{H}_{18} \mathrm{ON}_{2}$ requires $\mathrm{N}=10.07$ per cent.

\section{General Conclusions.}

(1) The homologues of coumarin may be converted into the corresponding thiocoumarins by treatment with phosphorus pentasulphide and these thiocoumarins, when purified from adherent sulphur compounds, exhibit a distinct yellow colour, in this respect differing from the coumarins which are colourless.

(2) Coumarin and its homologues and its sulphur analogues give rise to additive compounds with mercuric chloride in which the reagents combine in molecular proportion. In the case of the oxy- 
compounds, one of the following formulæ (III and View Article Online adopted.<smiles>Cl[Hg]C1CCCC1</smiles>

(III.)

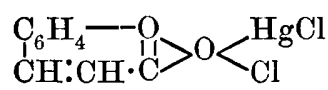

(IV.)<smiles>Cl[GeH](Cl)OC[Si]1CCCC1</smiles>

(V.)

(3) In the case of the thiocoumarins, it is probable that a formula of the former type is more appropriate. Thiocoumarin mercurichloride would thus have the constitution indicated by formula V. This view is confirmed by the fact that the thiocoumarins differ from the coumarins in their behaviour towards hydroxylamine and phenylhydrazine. These compounds, although regarded as agents for the detection of the carbonyl group, do not react with the coumarins (Tiemann, Ber., 1886, 19, 1663), whereas condensation does occur with the thiocoumarins, leading to the production of the coumarin oximes and the coumarinphenylhydrazones (VI and VII) :

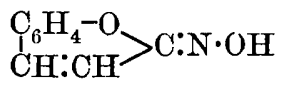

(VI.)

$$
\begin{aligned}
& \mathrm{C}_{6} \mathrm{H}_{4}-\mathrm{O} \\
& \mathrm{C} H: \mathrm{CH}
\end{aligned}>\mathrm{C}: \mathrm{N} \cdot \mathrm{NH} \cdot \mathrm{C}_{6} \mathrm{H}_{5} .
$$

(VII.)

(4) The negative results with the coumarins themselves support the view that in these lactones the ketonic group no longer exists as such, being modified in the sense indicated by formula VIII. On the other hand, the oxime and phenylhydrazone condensation which occurs with the thiocoumarins justifies the belief that the thiocompounds contain the thiocarbonyl group as represented by formula IX :

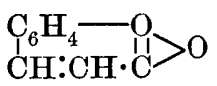

(VIII.)

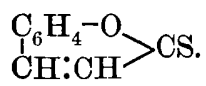

(IX.)

The marked colour difference between the coumarins and thiocoumarins may also be regarded as subsidiary evidence that they are differently constituted, for the coumarins are all colourless substances, whilst the thiocoumarins without exception have a distinctly yellow or orange colour.

The constitutional formulæ assigned to the additive compounds of the coumarins and thiocoumarins are based on one assumption, namely, that oxygen exhibits quadrivalency, more particularly towards the halogens or towards compounds containing the halogens. This supposition is in accordance with the general behaviour of oxygen and the halogens in both their inorganic and organic compounds.

Royal College of Science, London, South Kensingtox. 